\title{
Gender Differences in Neural Mechanisms Underlying Moral Judgment of Disgust: A Functional MRI Study
}

\author{
Juan Yang1,2*, Lili Guan ${ }^{1,2 *}$, Mingming Qi ${ }^{1,2}$ \\ ${ }^{1}$ Key Laboratory of Cognition and Personality, Ministry of Education, Southwest University, Chongqing, China \\ ${ }^{2}$ Department of Psychology, Southwest University, Chongqing, China \\ Email: valleyqq@swu.edu.cn
}

Received 22 March 2014; revised 28 April 2014; accepted 6 May 2014

Copyright $@ 2014$ by authors and Scientific Research Publishing Inc.

This work is licensed under the Creative Commons Attribution International License (CC BY). http://creativecommons.org/licenses/by/4.0/

(c) (i) Open Access

\section{Abstract}

Much recent research has sought to uncover the gender difference in neural mechanism of moral judgment; however, very few researches study the neural gender differences in a specific area of moral judgment. The aim of this functional magnetic resonance imaging (fMRI) study was to examine possible gender difference in neural response to (sexual) disgusting pictures versus neutral pictures. Seventeen participants ( 9 males) were scanned while viewing pictures of tactile intimacy in same-sex and being asked to evaluate whether the behaviors between the stimulus persons in the pictures were morally appropriate or not. Both the neural responses to pictures of tactile intimacy in same-sex between male participants and female participants and the neural response to pictures of male-male tactile intimacy and to pictures of female-female tactile intimacy were examined. The results showed that significantly increased differential activations to the disgusting pictures relative to the neutral pictures were observed in the dorsal medial prefrontal cortex (BA 9) and amygdala. Furthermore, greater activation to the pictures of female-female tactile intimacy was observed in the left superior frontal gyrus/dorsal medial prefrontal cortex (BA 6). These results suggested a possible neural gender difference between female's immoral behavior and male's immoral behavior.

\section{Keywords}

Gender Differences, Moral Judgment, Disgusting, Dorsal Medial Prefrontal Cortex

"Co-first author.

How to cite this paper: Yang, J., Guan, L.L. and Qi, M.M. (2014) Gender Differences in Neural Mechanisms Underlying Moral Judgment of Disgust: A Functional MRI Study. Journal of Behavioral and Brain Science, 4, 214-222. 


\section{Introduction}

The question of gender differences in moral judgment has been an issue of controversy and debate both in social psychology and in neuroscience. Kohlberg's moral judgment theory assumes that morality is universal, equal to male and female [1]. Other researchers question the possibility of a gender-related bias in Kohlberg's theory. Gilligan writes, "The very traits that traditionally have defined the 'goodness' of women, their care for and sensitivity to the needs of others, are those that mark them as deficient in moral development” [2]. She also describes two divergent modes of moral judgment: an ethic of care and an ethic of justice [3]. For males, they typically approach moral dilemmas in a rational way, with a justice-based orientation that respects rules and obligations. Whereas females are believed to typically approach moral dilemmas with a care-based orientation that emphasizes empathy and care for others. Despite substantial evidence confirming gender-related behavioral differences in moral judgment [4] [5], studies found no gender differences at all [6]. Researchers further have suggested that gender-related differences only exist in personal moral dilemma (those courses of action whose endorsement involved highly emotional decisions) where men give significantly more utilitarian answers than women do [7].

In the neuroimaging field, there are very few studies researched the gender differences in neutral mechanism underlying moral sensitivity except for Harenski et al. who have found a stronger relationship between posterior cingulated and insula activity during picture viewing and subsequent moral ratings in females and a stronger relationship between inferior parietal activity and moral ratings in males [8]. Other studies neglected to consider the gender factor, recruited subjects of one sex only [9] [10], or focused on sex differences in neural responses to pure disgusting emotions [11].

In the current study, we used functional magnetic resonance imaging (fMRI) to examine possible gender difference in brain activation to pictures depicting moral violations in healthy individuals. Moral violations were specific to tactile intimacy in same sex, which was rated as highly disgusting [12]. On one hand, neural responses to pictures of tactile intimacy in same sex between male participants and female participants would be examined. On the other hand, neural response to pictures of male-male tactile intimacy and to pictures of female-female tactile intimacy would also be examined.

The neural correlates of moral judgments within three moral areas: (physical) harm, dishonesty, and (sexual) disgust were investigated to test whether "moral judgment" was a unified category [13]. Across these studies, dorsal medial prefrontal cortex (dmPFC), which may represent the integration of emotional responses into moral decision-making was the only region activated by all scenarios judged to be morally wrong in comparison with neutral scenarios [13]. In addition, the specific roles of the ventromedial prefrontal cortex (vmPFC), medial prefrontal cortex (mPFC), anterior cingulate cortex (ACC), insula, amygdale, and posterior superior temporal suclus (pSTS) were also indicated in moral cognition [14].

Disgust is a universal basic emotion that functions to help protect an organism from ingesting potentially harmful substances, thereby promoting disease avoidance [15]. It has also been linked to moral judgments in many studies [10] [16]. The neural basis of disgust devoid of moral connotations ("pure disgust") and the experience of disgust disguised in the moral emotion of "indignation" were investigated and it was found that pure disgust and indignation recruited both overlapping and distinct brain regions, mainly in the frontal and temporal lobes [10]. In another study, the biological homology of pure disgust ("infection”) and moral disgust ("incest”) were investigated by using functional magnetic resonance imaging (fMRI). The results showed that even common brain regions were active during processing of both pure disgust and socio-moral acts; they still entrained unique brain regions [9]. Specifically, regions more activated in incest condition compared to the nonsexual moral condition were found in medial frontal gyrus and bilateral inferior frontal gyrus [9].

Functional neuroimaging study further uncovered the neural correlates of moral judgment in (sexual) disgust [13]. Results showed that the disgusting versus neutral scenario contrast revealed increased activity bilaterally in the dmPFC, amygdale, and posterior cingulate cortex (PCC). When normal adult volunteers passively viewed scenes evocative of basic (disgust and fear) and moral (compassion and indignation) emotions, significant bilateral activation occurred in the amygdale, thalamus, midbrain, and visual cortex [17].

In the current study, it was predicted that disgusting versus neutral pictures contrast would reveal increased activity bilaterally in the dmPFC and amygdale, which is consistent with previous study [13]. Further, males would show increased activity in brain regions associated with justice-based processing (superior temporal sulcus) relative to females [8]. In addition, given that females were traditionally expected to be peaceful and a 
care-based orientation in relations with others, females were more likely to exhibit intimate behavior such as embracing or kissing each other in daily life [18]. In other words, demonstrations of tactile intimacy between women might be viewed less disgusting than men. Therefore, compared to pictures of male-male tactile intimacy, pictures of female-female tactile intimacy would show activation in brain regions associated with the integration of emotional responses into moral decision-making (e.g., dmPFC).

\section{Methods}

\subsection{Ethics Statement}

The research has been approved by Southwest University ethics review board. After participants were given a complete explanation of the study, written informed consent was obtained from all of them.

\subsection{Participants}

Seventeen right-handed, healthy University students (9 male, aged 19-24 years; Mean $=22.4$ years, SD $=1.6$ ) participated in the study (Table 1). Participants did not have a history of psychiatric or neurological disorder, significant physical illness, head injury, or alcohol/drug abuse. All the participants were heterosexual (oral report). None of the participants were taking alcohol or medication at the time of the study. Participants were paid for their participation.

\subsection{Task}

The design was a $2 \times 2 \times 2$ factorial. The independent variables were the sex of the research participants (male or female), behavioral type (disgusting or neutral), and sex of the dyads shown in the picture (male or female). The behavioral type was based on whether the two stimulus figures were depicted as hugging and kissing each other or not. For disgusting pictures, the same-sex dyads were depicted as hugging and kissing each other. For neutral pictures, the same-sex dyads were depicted as standing face-to-face while looking and smiling at each other. The types of behavior in male-male pictures and female-female pictures were almost the same numbers. The stimuli were black-and-white photos of real people. The stimulus persons in the pictures were all Chinese and in their twenties. The pictures were plain because of the absence of any background (such as a street, a room, or the presence of other people), this lack of context encouraged participants to focus only on the stimulus persons when making their evaluation.

\subsection{Procedure}

In total, there were four types of stimuli in the experiment namely male-male disgusting pictures (MD), female-female disgusting pictures (FD), male-male neutral pictures (MN), and female-female neutral pictures (FN). The trials were presented in a randomized order in two functional runs and in each run, there were 15 trials for each type of stimuli. Stimulus appeared for $2 \mathrm{sec}$, after which they disappeared regardless of whether a response had been given or not. After each picture was displayed, the participants were asked to evaluate whether the behaviors between the stimulus persons in the pictures were morally appropriate or not and press two different buttons on a response button box according to their response (appropriate or inappropriate) in the scanner. The jitters between trials were $2 \mathrm{sec}, 4 \mathrm{sec}$, or 6 sec randomly and the screen remained white with a black fixation in the middle of the screen during the jitter.

The software package E-Prime (Psychology Software Tools Inc., Pittsburgh, PA) was used to deliver visual stimuli and record responses. Stimuli were projected onto a screen positioned at the end of the bore, visible through a mirror attached to the head-coil. Cushions were used to minimize head movement.

\subsection{Imaging Data Acquisition}

Images were acquired from a 3T Siemens TRIO MRI scanner. Functional data comprised 370 volumes acquired with T2 *-weighted gradient echo planar imaging (EPI) sequences. 32 echo planar images per volume sensitive to blood oxygenation level-dependent (BOLD) contrast were obtained (TR = $2000 \mathrm{msec}$; TE $=30 \mathrm{msec} ; 3 \times 3$ $\mathrm{mm}$ in-plane resolution; Field of View $[\mathrm{FOV}]=192$ ). Slices were acquired interleaved and oriented parallel to 
Table 1. Basic demographic characteristic of participants.

\begin{tabular}{cccc}
\hline & Males & Females & Total \\
\hline Sex & 9 & 8 & 17 \\
Age & $22.3(1.5)$ & $22.4(1.6)$ & $22.4(1.6)$ \\
\hline
\end{tabular}

the AC-PC plane, with thickness of $3 \mathrm{~mm}, 0.99 \mathrm{~mm}$ gap. High-resolution T1-weighted 3D fast-field echo (FFE) sequences were obtained for anatomical reference (176 slices, TR $=1900 \mathrm{msec}$; TE $=2.52 \mathrm{msec}$; slice thickness $=1 \mathrm{~mm} ; \mathrm{FOV}=250$; voxel size $=1 \times 1 \times 1 \mathrm{~mm}$ ).

\subsection{Imaging Data Analyses}

Data were analyzed using the Brain Voyager QX v2.0 software (Brain Innovation, The Netherlands). Due to T1 saturation effects, the first 2 scans of each run were discarded from the analysis. Preprocessing of functional scans included slice-scan time correction using 'cubic spine' interpolation. Data were motion corrected to minimize effects of head motion on analyses. To improve signal detection, temporal and spatial filtering was applied. Drift removal was done using a high-pass temporal filter (3cycles/run, equivalent to 0.008 Hz). Spatial smoothing was achieved by applying a 4-mm full-width at half maximum Gaussian filter. Spatial normalization was performed for structural and functional data sets. Spatial normalization of the structural volumes was performed in two steps. The first step consisted in aligning the 3D MPRAGE data set of each subject with the stereotaxic axes. For this step the location of the anterior commissure (AC), the posterior commissure (PC) and two rotation parameters for mid-sagittal alignment had to be specified manually in the 3D data set. In the second step the extreme points of the cerebrum were specified. These points together with the AC and PC coordinates were then used to scale the 3D data sets into the dimensions of the standard brain of the atlas of Talairach and Tournoux using a piecewise affine and continuous transformation.

To transform the functional data into Talairach space, the preprocessed functional time series were first resampled at a voxel size of $3 \mathrm{~mm} \times 3 \mathrm{~mm} \times 3 \mathrm{~mm}$ and coregistered with the corresponding structural data set. The co-registration transformation in Brain Voyager QX was determined concatenating an initial alignment matrix obtained using the Siemens position parameters of the functional and structural images with a fine-tuning alignment matrix obtained by means of an intensity-driven alignment algorithm. The alignment between functional and anatomical scans was finally checked by means of an accurate visual inspection. Then the rigid-body AC-PC transformation performed for the 3D anatomical data set was applied to co registered functional data. This procedure resulted in a normalized four-dimensional data representation (volume time course) for each functional run.

In order to avoid quality loss due to successive data sampling, the spatial normalization was actually performed using a single transformation matrix obtained combining the different spatial transformations described. Analyses of the time series data were performed on individual participants using an event-related model convolved with the canonical homodynamic response function. The multiple regression analysis implementing a model of expected BOLD signal changes for each of four trial categories (MD, FD, MN and FN) and the null event was performed for each subject. Applying linear contrasts to the obtained parameter estimates, effects of interest were tested across all the subjects (random effects model). The resultant single participant images were then entered into second-level ANOVA analyses with two within-subjects factors (behavioral type and sex of the dyads shown in the picture) and one between subjects factor (sex of the research participants). To further extract areas showing a significant main effect of behavioral type, contrasts (MD + FD)-(MN + FN) (disgust > neutral) and $(\mathrm{MN}+\mathrm{FN})-(\mathrm{MD}+\mathrm{FD})$ (neutral > disgust) were tested. To extract areas showing a significant main effect of sex of the dyads shown in the picture, (MD + MN)-(FD + FN) (male > female) and (FD + FN)-(MD + $\mathrm{MN}$ ) (female $>$ male) were tested. Inference of statistical significance of these analyses used uncorrected $P$ value at least $P<0.001$, in regions encompassing at least 4 voxels.

\section{Results}

\subsection{Behavioral Data}

Moral judgments of male disgusting pictures (MD), female disgusting pictures (FD), male neutral pictures (MN) 
and female neutral pictures (FN) both in the male and female participants were listed in Figure 1. Both participants groups judged $80 \%$ of the disgusting pictures as morally inappropriate. Repeated-measure ANOVA showed that the interaction effect of behavioral type (disgusting or neutral) by sex of the dyads shown in the picture (male or female) by sex of research participants was significant, $F(1,15)=5.65, p<0.05$. Male participants evaluated less MD pictures as inappropriate than FD pictures, $p<0.05$ and male participants evaluated less FD pictures as inappropriate than female participants did, $p<0.05$. The reaction times of male and female participants to the MD, FD, MN and FN pictures were listed in Table 2. Repeated-measure ANOVA showed that there was a significant effects of sex of dyads shown in the picture (male or female), $F(1,15)=38.405, p<$ 0.001 . The female pictures were responded significantly slower than the male pictures. There was also a significant effects of sex of research participants, $F(1,15)=14.33, p<0.01$. It took much more time for the male participants than for the female participants to make the decisions. The interaction effect of behavioral type (disgusting or neutral) by sex of the dyads shown in the picture (male or female) was significant, $F(1,15)=10.748, p<0.01$. The pictures in the FD condition were responded significantly slower than in the MD condition, $p<0.01$.

\subsection{Functional Imaging Results}

The main effect and the interaction effects ( $F$ tests) of the ANOVA analyses were described as below. The main effect of behavioral type (disgusting vs. neutral) was observed in the dorsal medial prefrontal cortex (BA9). Additional activations were observed in amygdala. The interaction effect of behavioral type (disgusting vs. neutral) by sex of the dyads shown in the picture (male or female) was observed in the superior frontal gyrus/dorsal medial prefrontal cortex (BA6). There was not a main effect or interaction effect of sex of the research participants.

Areas showing a significant main effect of behavioral type were given in Table 3 and Figure 2(a). Significantly increased differential activations to the disgusting pictures relative to the neutral pictures were observed in the dorsal medial prefrontal cortex (BA9) and amygdala. No significant differential activation to the neutral pictures compared with the disgusting pictures was observed.

Areas showing a significant main effect of sex of dyads shown in the pictures were given in Table 2 and

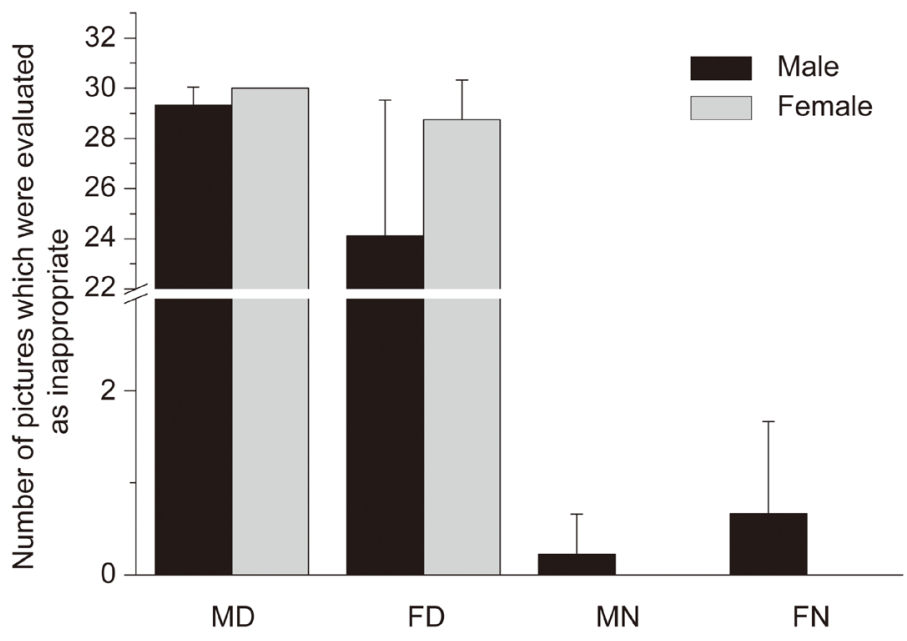

Figure 1. Moral judgments of male disgusting pictures (MD), female disgusting pictures (FD), male neutral pictures (MN) and female neutral pictures (FN) both in the male and female participants.

Table 2. The mean reaction time (msec) of male and female participants to each type of pictures.

\begin{tabular}{rcccc}
\hline & MD & FD & MN & FN \\
\hline Male & $972(88)$ & $1102(117)$ & $1002(129)$ & $1052(148)$ \\
Female & $798(82)$ & $915(82)$ & $875(94)$ & $887(120)$ \\
\hline
\end{tabular}

MD: male disgusting picture; MN: male neutral picture; FD: female disgusting picture; FN: female neutral picture. 
Table 3. Areas showing a significant main effect of behavioral type and sex of dyads.

\begin{tabular}{|c|c|c|c|}
\hline Structure & Talairach coordinate $(\mathrm{x}, \mathrm{y}, \mathrm{z})$ & $t$ value & cluster size \\
\hline \multicolumn{4}{|l|}{ Disgusting $>$ Neutral } \\
\hline Dorsal medial prefrontal cortex & $-5,54,22$ & 7.92 & 634 \\
\hline Amygdala & $-20,-5,-9$ & 8.96 & 227 \\
\hline \multicolumn{4}{|l|}{ Neutral > Disgusting } \\
\hline \multicolumn{4}{|l|}{ No significant activation } \\
\hline \multicolumn{4}{|l|}{ Female $>$ male } \\
\hline Dorsal lateral prefrontal cortex & $2,18,48$ & 6.79 & 91 \\
\hline \multicolumn{4}{|l|}{ Male $>$ female } \\
\hline \multicolumn{4}{|l|}{ No significant activation } \\
\hline \multicolumn{4}{|l|}{$\begin{array}{c}\text { Female-disgusting > } \\
\text { Male-disgusting }\end{array}$} \\
\hline Dorsal medial prefrontal cortex & $-9,20,50$ & 7.72 & 94 \\
\hline
\end{tabular}
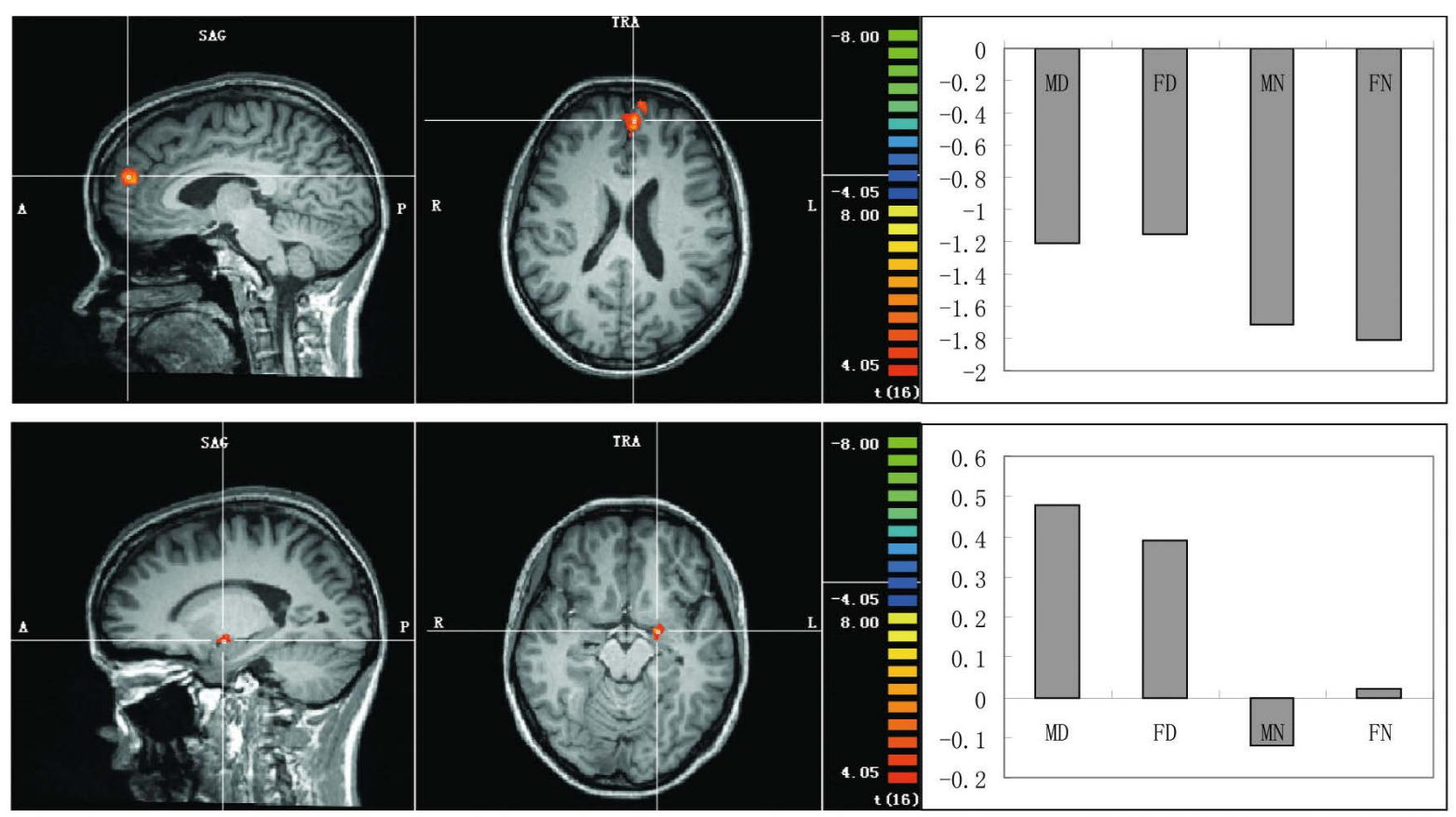

(a)
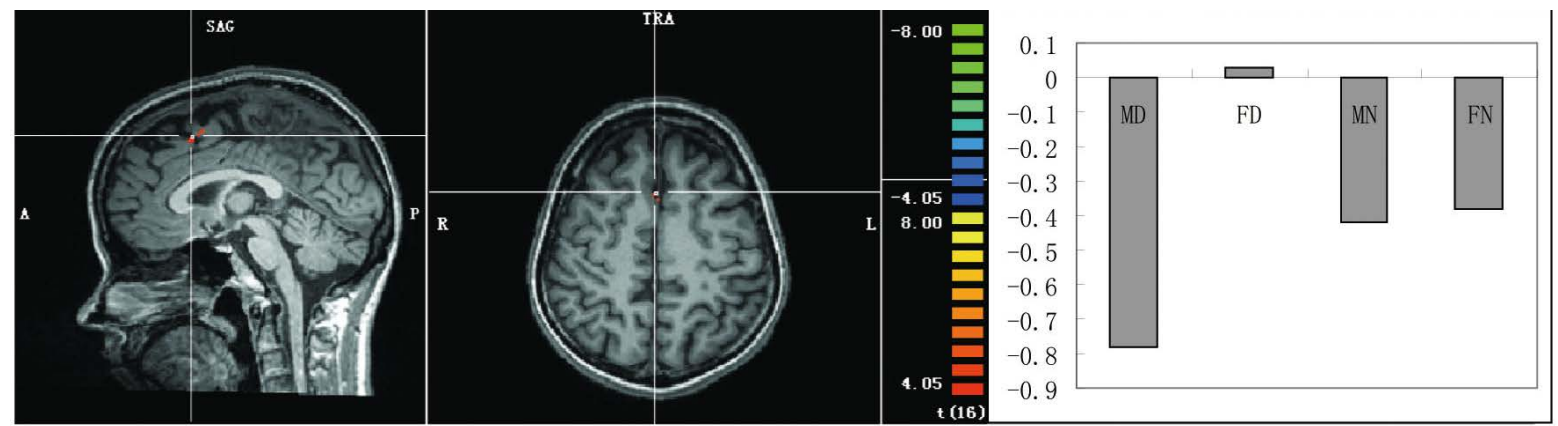

(b) 


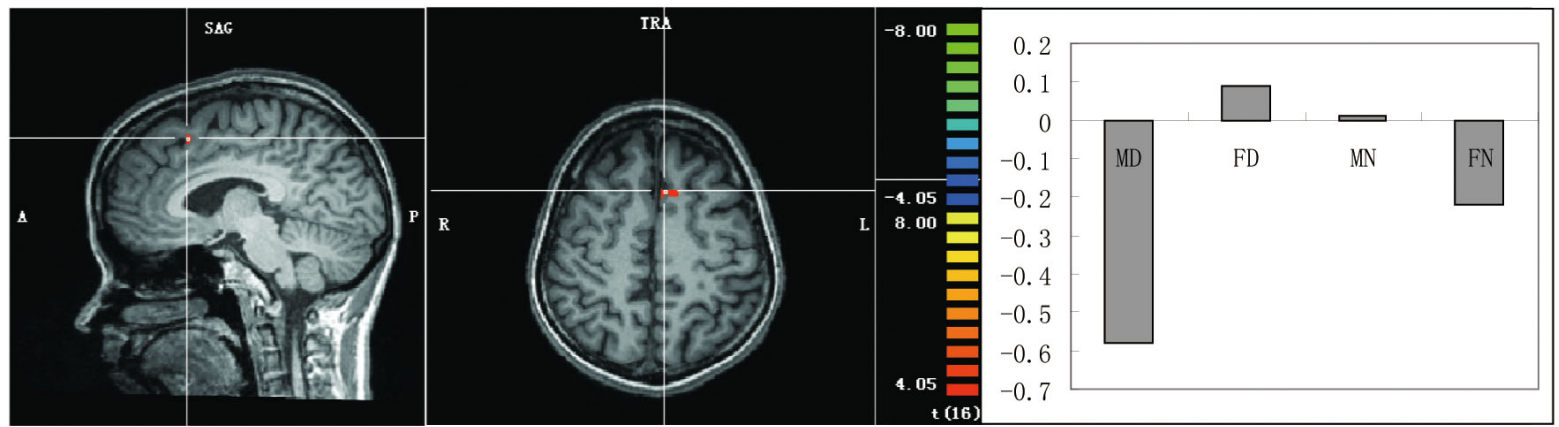

(c)

Figure 2. (a) Areas showing greater activation to the disgusting pictures. (b) An area showing a significant main effect of sex of dyads shown in the pictures with greater activation in the female pictures than the male pictures. (c) An area showing a significant interaction with greater activation to the FD pictures. Representative activated areas are superimposed on an appropriate slice of the mean normalized anatomical T1-weighted MRI. Activation profile in the parameter estimates is also shown for each of these activation peaks.

Figure 2(b). Significantly increased differential activations to the female pictures relative to the male pictures were observed in the right superior frontal gyrus and dorsal lateral prefrontal cortex (BA8). No significant differential activation to the male pictures compared to the female pictures was observed.

A significant interaction with greater activation to the FD pictures was observed in the left superior frontal gyrus/dorsal medial prefrontal cortex (BA6) (Table 2 and Figure 2(c)). In this area, no significant difference in the activation pattern was observed between $\mathrm{MN}$ and FN; therefore, the differential activation in this area was specific for the FD pictures.

\section{Discussion}

The purpose of the current study was to investigate possible gender difference in brain activation to pictures depicting moral violations in healthy individuals. Some of the hypothesis was confirmed, as significantly increased differential activations to the disgusting pictures relative to the neutral pictures were observed in the dorsal medial prefrontal cortex and amygdala. Furthermore, greater activation to the female disgusting (FD) pictures was observed in the left superior frontal gyrus/dorsal medial prefrontal cortex. However, some of the hypothesis was not confirmed, as there was no gender difference of participant in brain activation to moral judgment of tactile intimacy in same-sex.

Although researchers have proposed that females and males differ in the structure of their moral attitudes, such that females tend to adopt care-based moral evaluations and males tend to adopt justice-based moral evaluations [2] [3], and neuroimaging results have further suggested gender differences in strategies utilized in moral appraisals [8], other study still have concluded that gender differences in moral judgment specifically influence personal moral dilemmas involving emotionally salient actions in which males have significantly more utilitarian choices [7]. In the current study, the moral judgment stimuli were the tactile intimacy in same-sex which was not as emotionally salient as in previous study. Therefore, it would be no surprise that there was no gender difference of participant in brain activation to moral judgment of tactile intimacy in same-sex.

Further, given that males are traditionally expected to be competitive and achievement-oriented in relations with other men, demonstrations of tactile intimacy between men might be viewed more negatively [18]. Also, men, as compared to women, have less positive attitudes toward same-sex touching than women do [19]. In other words, the moral judgment task in the current study led to a bias that both male and female participants would judge tactile intimacy between males more disgusting. This would be another reason of missing gender difference of participant in brain activation to moral judgment of tactile intimacy in same-sex. Finally, the small numbers of the participants would be the other reason to detect such an effect.

It was found that the judgment of moral wrongness was sub-served by distinct neural systems for each of the different moral areas [9] [13]. Dishonest, disgusting, and harmful moral transgression recruited networks of brain regions associated with mentalizing, affective processing, and action understanding, respectively. Although researchers have tried to uncover the neural basis of moral judgment, there has been no study to investi- 
gate gender differences in neural mechanisms underlying moral sensitivity within three moral areas: (physical) harm, dishonesty, and (sexual) disgust, which would be the future direction of this field.

Compared to the neutral pictures, disgusting pictures activated the dmPFC and amygdala, which was consistent with previous study and our hypothesis [9] [13] [20]. Amygdala, a structure commonly associated with fear processing, can also be preferentially involved in disgusting processing [9]. The disgusting versus neutral scenario contrast also revealed increased activity bilaterally in the amygdale [13]. Across moral judgment studies, activation of dorsal medial PFC, an area implicated in integration of emotional responses into moral decisionmaking was most widely associated with morally relevant processing [13]. Researchers have suggested that emotional responses and cognitive computations work in tandem to give rise to moral judgments [21] [22]. Notably, an emotional learning system mediated by the amygdale and system for decision making on the basis of reinforcement expectations mediated by the medial orbitofrontal cortex is crucial for cueing morally appropriate behavior and the acquisition of moral knowledge during childhood [20]. Research has found that disgusting transgressions were associated with increased bilateral activity in the amygdale, and dmPFC, areas implicated in social-emotional processing [13].

Our results further found that greater activation to the female disgusting (FD) pictures was observed in the left superior frontal gyrus/dorsal medial prefrontal cortex. Activation of the dorsal medial prefrontal cortex, an area implicated in thinking about others and self-referential processing [23] [24], especially in ambiguous circumstances [25], is most widely associated with morally relevant processing [14]. First of all, activity in this region is modulated by ambiguity both in social and nonsocial contexts [25]. In the current study, since the females were more likely to exhibit intimate behavior, it was more ambiguous for participants to evaluate whether their behaviors were morally appropriate when both females had tactile intimacies. Thus, the processing of ambiguous information represented one explanation for the dmPFC activation to the female disgusting pictures. Secondly, this area is consistently activated when thinking about other people and in self-referential processing [23] [24]. It is likely that the evaluation of female disgusting pictures involved a deeper evaluation between people's motives and actions shown in the picture and participants' own values. Thirdly, this area represents the integration of emotional responses into moral decision-making [13]. Since females were more likely to exhibit intimate behavior such as embracing or kissing each other in daily life and demonstrations of tactile intimacy between women might be viewed less disgusting than men [18], it would be more difficult for participants to integrate disgusting emotion into moral cognition for female disgusting pictures than male disgusting pictures. Finally, even the current results showed that greater activation to the female disgusting (FD) pictures was observed in the left superior frontal gyrus/dorsal medial prefrontal cortex; future study should investigate this phenomenon in the other two moral areas: (physical) harm and dishonesty.

A number of limitations are present in the current study. Firstly, the small samples sum is not very strong validation about this hypothesis, future study should increase the amount of subjects to increase statistical power. Secondly, participants were asked to evaluate whether the behaviors between the persons in the pictures were morally appropriate or not, which could be an uncertain question for participants. Another good way to explore this issue might be asking participants to determine whether it represented a moral violation or was morally wrong.

\section{Acknowledgements}

This work was supported by the Fundational Research Funds for the Central Universities (SWU1309105). We would like to thank Mingming Qi for assistance with MRI scanning.

\section{References}

[1] Kohlberg, L. (1964) Development of Moral Character and Moral Ideology. In: Hofmann, M.L., hOfmann, L.W., Eds., Review of Child Development Research. Russell Sage Foundation, New York.

[2] Gilligan C. (1982) In a Different Voice: Psychological Theory and Women’s Development. Harvard University Press, Cambridge.

[3] Gilligan C. (1977) In a Different Voice: Women's Conceptions of Self and of Morality. Harvard Educational Review, 47, 481-517.

[4] Bjorklund F. (2003) Differences in the Justification of Choices in Moral Dilemmas: Effects of Gender, Time Pressure and Dilemma Seriousness. Scandinavian Journal of Psychology, 44, 459-466. 
http://dx.doi.org/10.1046/j.1467-9450.2003.00367.x

[5] Aldrich, D. and Kage, R. (2003) Mars and Venus at Twilight: A Critical Investigation of Moralism, Age Effects, and Sex Differences. Political Psychology, 24, 23-40. http://dx.doi.org/10.1111/0162-895X.00315

[6] Jaffee, S. and Hyde, JS. (2000) Gender Differences in Moral Orientation: A Meta-Analysis. Psychological Bulletin, 126, 703-726. http://dx.doi.org/10.1037/0033-2909.126.5.703

[7] Fumagalli, M., Ferrucci, R., Mameli, F., Marceglia, S., Mrakic-Sposta, S., Zago, S., et al. (2010) Gender-Related Differences in Moral Judgments. Cognitive Processing, 11, 219-226. http://dx.doi.org/10.1007/s10339-009-0335-2

[8] Harenski, C.L., Antonenko, O., Shane, M.S. and Kiehl, K.A. (2008) Gender Differences in Neural Mechanisms Underlying Moral Sensitivity. Social Cognitive and Affective Neuroscience, 3, 313-321. http://dx.doi.org/10.1093/scan/nsn026

[9] Borg, J.S., Lieberman, D. and Kiehl, K.A. (2008) Infection, Incest and Iniquity: Investigating the Neural Correlates of Disgust and Morality. Journal of Cognitive Neuroscience, 20, 1529-1546. http://dx.doi.org/10.1162/jocn.2008.20109

[10] Moll, J., de Oliveira-Souza, R., Moll, F.T., Ignacio, F.A., Bramati, I.E., Caparelli-Daquer, E.M., et al. (2005) The Moral Affiliations of Disgust: A Functional MRI Study. Cognitive and Behavioral Neurology, 18, 68-78. http://dx.doi.org/10.1097/01.wnn.0000152236.46475.a7

[11] Caseras, X., Mataix-Cols, D., An, S.K., Lawrence, N.S., Speckens, A., Giampietro, V., et al. (2007) Sex Differences in Neural Responses to Disgusting Visual Stimuli: Implications for Disgust-Related Psychiatric Disorders. Biological Psychiatry, 62, 464-471. http://dx.doi.org/10.1016/j.biopsych.2006.10.030

[12] Kozloski, M.J. (2010) Homosexual Moral Acceptance and Social Tolerance: Are the Effects of Education Changing? Journal of Homosexuality, 57, 1370-1383. http://dx.doi.org/10.1080/00918369.2010.517083

[13] Parkinson, C., Sinnott-Armstrong, W., Koralus, P.E., Mendelovici, A., McGeer, V. and Wheatley, T. (2011) Is Morality Unified? Evidence that Distinct Neural Systems Underlie Moral Judgments of Harm, Dishonesty, and Disgust. Journal of Cognitive Neuroscience, 23, 3162-3180. http://dx.doi.org/10.1162/jocn_a_00017

[14] Moll, J., Zahn, R., de Oliveira-Souza, R., Krueger, F. and Grafman, J. (2005) Opinion: The Neural Basis of Human Moral Cognition. Nature Reviews Neuroscience, 6, 799-809. http://dx.doi.org/10.1038/nrn1768

[15] David, B. and Olatunji, B.O. (2011) The Effect of Disgust Conditioning and Disgust Sensitivity on Appraisals of Moral Transgressions. Personality and Individual Differences, 50, 1142-1146. http://dx.doi.org/10.1016/j.paid.2011.02.004

[16] Haidt, J., McCauley, C. and Rozin, P. (1994) Individual Differences in Sensitivity to Disgust: A Scale Sampling Seven Domains of Disgust Elicitors. Personality and Individual Differences, 16, 701-713. http://dx.doi.org/10.1016/0191-8869(94)90212-7

[17] Moll, J., de Oliveira-Souza, R., Bramati, I.E. and Grafman, J. (2002) Functional Networks in Emotional Moral and Nonmoral Social Judgments. Neuroimage, 16, 696-703. http://dx.doi.org/10.1006/nimg.2002.1118

[18] Derlega, V.J., Lewis, R.J., Harrison, S., Winstead, B. and Costanza, R. (1989) Gender Differences in the Initiation and Attribution of Tactile Intimacy. Journal of Nonverbal Behavior, 13, 83-96. http://dx.doi.org/10.1007/BF00990792

[19] Derlega, V.J., Catanzaro, D. and Lewis, R.J. (2001) Perceptions about Tactile Intimacy in Same-Sex and Opposite-Sex Pairs Based on Research Participants’s Sexual Orientation. Men Masculinity, 2, 124-32. http://dx.doi.org/10.1037/1524-9220.2.2.124

[20] Decety, J., Michalska, K.J. and Kinzler, K.D. (2011) The Contribution of Emotion and Cognition to Moral Sensitivity: A Neurodevelopmental Study. Cereb Cortex.

[21] Dupoux, E. and Jacob, P. (2007) Universal Moral Grammar: A Critical Appraisal. Trends in Cognitive Sciences, 11, 373-378. http://dx.doi.org/10.1016/j.tics.2007.07.001

[22] Greene, J.D., Nystrom, L.E., Engell, A.D., Darley, J.M. and Cohen, J.D. (2004) The Neural Bases of Cognitive Conflict and Control in Moral Judgment. Neuron, 44, 389-400. http://dx.doi.org/10.1016/j.neuron.2004.09.027

[23] Amodio, D.M. and Frith, C.D. (2006) Meeting of Minds: The Medial Frontal Cortex and Social Cognition. Nature Reviews Neuroscience, 7, 268-277. http://dx.doi.org/10.1038/nrn1884

[24] Northoff, G., Heinzel, A., de Greck, M., Bermpohl, F., Dobrowolny, H. and Panksepp, J. (2006) Self-Referential Processing in Our Brain-A Meta-Analysis of Imaging Studies on the Self. Neuroimage, 31, 440-457. http://dx.doi.org/10.1016/j.neuroimage.2005.12.002

[25] Jenkins, A.C. and Mitchell, J.P. (2010) Mentalizing under Uncertainty: Dissociated Neural Responses to Ambiguous and Unambiguous Mental State Inferences. Cereb Cortex, 20, 404-410. http://dx.doi.org/10.1093/cercor/bhp109 